\title{
Videolaryngoscope-guided intubation without neuromuscular blockade in a patient with Huntington's disease
}

\author{
Hyun Young Lee ${ }^{1,2}$, Ki Tae Jung ${ }^{1,2}$, Su Yeun Cho', Sang Hun Kim ${ }^{1,2}$ \\ Department of Anesthesiology and Pain Medicine, ${ }^{1}$ Chosun University Hospital, ${ }^{2}$ Chosun University School of Medicine, Gwangju, \\ Korea
}

Received May 28, 2020

Revised June 10, 2020

Accepted June 10, 2020

Corresponding author

Sang Hun Kim

Department of Anesthesiology and

Pain Medicine, Chosun University

School of Medicine, 309 Pilmun-

daero, Dong-gu, Gwangju 61453,

Korea

Tel: + 82-62-220-3223

Fax: + 82-62-223-2333

E-mail:ksh3223@chosun.ac.kr

ORCID:

https://orcid.org/0000-0003-3869-9470
Huntington's disease (HD) has a risk of potential perioperative complications such as aspiration, drug interactions with anesthetics, agitation, psychosis, shivering, and spasms. Thus, inexperienced anesthesiologists may face challenges in the management of HD patients. A 54-yearold man with HD was scheduled to undergo open reduction and internal fixation of an intertrochanteric femur fracture. We successfully performed videolaryngoscope-guided intubation after propofol sedation and oral topical anesthesia, as awake fibreoptic bronchoscopy-guided intubation had failed because of noncooperation and choreiform movements. Total intravenous anesthesia was maintained with propofol and remifentanil infusion, and intraoperative neuromuscular block was controlled with rocuronium and sugammadex successfully, without any postoperative complications. His psychotropic medications were restarted from the morning of postoperative day 1. Videolaryngoscope-guided intubation, total intravenous anesthesia, use of rocuronium and sugammadex, and re-administration of psychotropic medication as soon as possible form one of the successful regimens for HD patients.

Keywords: Huntington disease; Intravenous anesthesia; Laryngoscopy; Propofol; Rocuronium; Sugammadex

\section{INTRODUCTION}

Huntington's disease (HD) is a rare autosomal dominant disorder caused by a mutation in the Huntingtin gene on chromosome 4, and its onset is typically between 30 and 50 years of age [1,2]. This Huntingtin gene has considerable inter-individual variability in the length of cytosine-adenine-guanine (CAG) trinucleotides, and CAG repeats exceeding the normal limit of 35 repeats have been correlated with the progression of motor and cognitive deficits [1,2]. $\mathrm{HD}$ is characterized by the triad of motor, neuropsychiatric and cognitive disturbances, which presents with symptoms of chorea, dystonia, hypokinesia, ataxia, cognitive decline, and behavioral difficulties $[1,3]$. With disease progression, dysphagia with dysfunction of pharyngeal muscles develops, increasing the risk of aspiration [1,3]. In addition, an exaggerated response and an increased sensitivity to anesthetics, prolonged intraoperative apnea and recovery, postoperative agitation and psychosis, intense postoperative shivering, and generalized tonic spasm have been suggested as potential perioperative complications in HD patients [2,4]. However, with the low prevalence of $\mathrm{HD}$, there is a paucity 
of literature on the anesthetic management of HD patients. Therefore, the inexperienced anesthesiologist may face challenges in the management of HD patients, and ideal anesthetic management regimens are yet to be determined. Herein, we present a case report of the successful anesthetic management of a HD patient under total intravenous anesthesia after videolaryngoscope-guided intubation with propofol sedation and oral topical anesthesia, along with a review of literature.

\section{CASE REPORT}

This case report was approved by the Institutional Review Board of Chosun University Hospital (February, 4th, 2020; IRB number: Chosun 2020-01-020), and written informed consent was obtained from a patient and a legal surrogate. The 54-year-old, 56-kg male patient was scheduled to undergo an open reduction and internal fixation for an intertrochanteric femur fracture. He was diagnosed with $\mathrm{HD}$ with 44 repeats of Huntingtin alleles 4 years ago, which was complicated by chorea, dystonia, dementia, and depression. He took psychotropic medications, which consisted of oral haloperidol $10 \mathrm{mg}$ BID, alprazolam $1 \mathrm{mg}$ BID, escitalopram $10 \mathrm{mg}$ QD, donepezil $10 \mathrm{mg}$ QD, and clonazepam $1 \mathrm{mg}$ BID. These medications were discontinued on the morning of the surgery by a neurologist. Preanesthetic evaluation showed results within normal limits except for a T-wave abnormality in the electrocardiogram (ECG), increased aspartate transaminase/alanine transaminase (63.2/59.3 U/L), increased creatine kinase $(928 \mathrm{U} / \mathrm{L}$ ), and anemia (hemoglobin: $7.9 \mathrm{~g} /$ dL). Hemoglobin was restored to $10.8 \mathrm{~g} / \mathrm{dL}$ after transfusion of 1 unit packed red blood cells, and other abnormal laboratory results were controlled with conservative management. The preanesthetic evaluation of the airway was normal with a Mallampati Class I, thyromental distance, adequate mouth opening, and neck range of motion. After premedication with low dose intramuscular midazolam (1.5 mg)because he did not show swallowing dysfunction and had preoperative anxiety-he was transported to the operating room. Prior to the induction of anesthesia, standard patient monitoring devices to obtain ECG, non-invasive blood pressure, end-tidal partial pressure of carbon dioxide $\left(\mathrm{ETCO}_{2}\right)$, and peripheral oxygen saturation $\left(\mathrm{SpO}_{2}\right)$ were applied. The initial values were as follows: blood pressure, 139/75 mmHg; heart rate, 61 beats/min with sinus rhythm; $\mathrm{SpO}_{2}, 98 \%$. In addition, the TOF-Scan ${ }^{\text {TM }}$ (IDMED, France) and the SEDline monitor (Sedline Inc., USA) were applied for assessment of the depth of neuromuscular blockade as well as of anesthesia. We planned an awake fibreoptic bronchoscopy-guided intubation (FOBI) to prevent aspiration as the first airway management. He was pre-anesthetized with $10 \%$ xylocaine spray on the tongue base and oral cavity, and pre-oxygenated with $\mathrm{O}_{2}$ at $6 \mathrm{~L} / \mathrm{min}$ via a facial mask for $5 \mathrm{~min}$. However, the FOBI was difficult because of noncooperation and choreiform movements. Therefore, in the next stage after failure of two FOBI attempts, a videolaryngoscope-guided intubation (VLI) with GlideScope (Verathon Inc., USA) under Sellick's maneuver was performed cautiously and gently under previous anesthesia on the tongue base and oral cavity, at the patient state index (PSI) of SEDline score $<60$ with target-controlled intravenous infusion of propofol $(2 \mu \mathrm{g} / \mathrm{mL})$. After successful VLI, remifentanil $(2 \mathrm{ng} / \mathrm{mL})$ was co-administrated with the target-controlled infusion system and a single dose of rocuronium $(0.5 \mathrm{mg} / \mathrm{kg})$ was injected. The intraoperative infused propofol concentrations were adjusted to maintain the PSI between 30 and 60. The intraoperative infused remifentanil concentrations were adjusted within $\pm 20 \%$ of mean arterial pressure changes. The intraoperative vital signs were stable. The settings of mechanical ventilation were also adjusted to maintain the $\mathrm{ETCO}_{2}$ between 32 and $42 \mathrm{mmHg}$ with a 50\% oxygen-air mixture for $100 \mathrm{~min}$. A single dose of rocuronium (20 mg) was injected for maintenance of at least 2 twitches of train-of-four test (TOF). The forced-air convective warming device was not applied to maintain the intraoperative body temperature because he had mild hyperthermia $\left(37.2^{\circ} \mathrm{C}\right)$. At the end of surgery, the distal esophageal temperature was $36.9^{\circ} \mathrm{C}$ and the total infused doses of propofol and remifentanil were $752 \mathrm{mg}$ and $378 \mu \mathrm{g}$, respectively. He was transferred to the recovery room after extubation at TOF score above 0.9 with the injection of sugammadex $(200 \mathrm{mg})$. Postoperative pain was controlled with intravenous patient-controlled analgesia (PCA) consisting of fentanyl 1,000 $\mu$, nefopam $120 \mathrm{mg}$, and ramosetron $1.2 \mathrm{mg}$ for 2 days. The setting of PCA device was total volume, $100 \mathrm{~mL}$; basal infusion rate, $2 \mathrm{~mL} / \mathrm{h}$; bolus volume, $2 \mathrm{~mL}$; and lock-out time, $10 \mathrm{~min}$. His prescribed psychotropic medications were restarted from the morning of postoperative day 1 . He was discharged without postop- 
eratively aggravated HD symptoms and complications on postoperative day 7 .

\section{DISCUSSION}

The primary anesthetic management of $\mathrm{HD}$ patients usually focuses on the prevention of $\mathrm{HD}$ symptom aggravation, perioperative airway protection, and a safe and rapid postoperative recovery [5]. Firstly, HD patients usually take psychotropic medications, such as antidepressants, benzodiazepine, antipsychotics, and antiepileptics [1,3]. It is important to decide whether these medications should be continued during perioperative period or stopped before anesthesia, considering the drug-interactions with anesthetics. General anesthesia may result in postoperative agitation, chorea, and psychosis because of its potential to exacerbate neuropsychiatric symptoms [4]. Hence, some authors have suggested that the perioperative cessation of these medications can aggravate the symptoms of HD and they need to be continued [6]. However, we consider the risk of postoperative worsening of abnormal movements to be the result of the skipped morning dose of HD medication [7]. Therefore, psychotropic medications should be administered as soon as possible after surgery [7]. In this case, we discontinued the patient's psychotropic medications on the morning of surgery according to the preoperative recommendation of a neurologist, and these medications were successfully restarted without any exacerbation of neuropsychiatric symptoms.

Secondly, advanced HD patients have a risk of regurgitation and aspiration due to dysphagia with dysfunction of pharyngeal muscles $[2,8,9]$. Therefore, the awake FOBI is recommended for prevention of these complications [10]. As another option, rapid sequence induction with cricoid pressure can be applied in $\mathrm{HD}$ patients with aspiration risk [2,6,11]. However, TOF monitoring is essential throughout the operation, because inappropriate neuromuscular relaxation can cause gag reflex, and the residual paralysis after recovery can cause respiratory problems along with aspiration [2]. Therefore, if awake intubation is not required, intubation and extubation should be performed when the TOF ratio is 0 and above 0.9 , respectively [2]. In addition, some authors suggested that endotracheal intubation could also be successfully performed without neuromuscular blockade under sedoanalgesia with propofol, lidocaine, and opioid [12]. Traditional endotracheal intubation can also be performed safely in early HD patients if the "nil per os" is sufficient, because there is no evidence of impaired gastric emptying in these patients [13]. However, in this case, HD was diagnosed 4 years ago, and dysfunction of pharyngeal muscles was not completely excluded, even though there was no evidence of aspiration and regurgitation risk. Therefore, we used the awake FOBI as the first option, but it failed because of noncooperation and choreiform movements in an awake state. As the second option, we performed VLI with cricoid pressure after propofol sedation without the co-administration of neuromuscular blockade and opioid, because the airway of this patient was already anesthetized enough with the $10 \%$ xylocaine spray. VLI was done successfully without any complications such as gag reflex, regurgitation, aspiration, or hypoxemia.

Thirdly, the perioperative anesthetics should be chosen carefully considering the risks of prolonged response and increased sensitivity to anesthetics $[2,4,8,9]$. For neuromuscular blockade, rocuronium is commonly recommended in $\mathrm{HD}$ patients, even though atracurium and mivacurium can also be used safely, because rocuronium can be completely reversed with sugammadex $[2,9,11,14]$. However, succinylcholine is considered avoidable because of the prolonged neuromuscular block in HD patients with low plasma cholinesterase levels, except in patients with a potential risk of aspiration [2]. As anesthetics for anesthesia induction and maintenance, intravenous or inhalational anesthetics, sedatives, and hypnotics can also be used safely without specific contraindications in HD patients [2,4,9,10,14,15]. However, we should consider that inhalational anesthetics, sedatives, and hypnotics have the risk of generalized muscle spasms, prolonged shivering, or protracted recovery periods theoretically [8]. Hence, some authors recommended that total intravenous anesthesia (TIVA) using propofol and remifentanil is useful from the point of view of the precise titration, ease of induction, and a rapid, controlled recovery with their specific pharmacokinetics [8,10]. Propofol also has advantages such as ceasing of choreiform movements and a rapid recovery from anesthesia, $[8,10,15]$ while it has the risk of postoperative worsening of abnormal movements by its neuroexcitation effect [7]. However, this propofol-induced neuroexcitation can be mitigated by the perioperative continuation or early re-administration after surgery of pre- 
operative psychotropic medications [15]. In this case, even though we used TIVA with propofol and remifentanil, exacerbated neuropsychiatric symptoms, postoperative agitation, and other complications were not found after anesthesia because of the short surgical time and the re-initiation of $\mathrm{HD}$ medication as soon as possible. As a reversal agent, if anticholinergics are required for control of hypersecretion and for neuromuscular blockade reversal, glycopyrrolate is preferred to atropine because the central anticholinergic effects of atropine may exacerbate choreiform movements [5,12]. In this case, we did not premedicate with anticholinergics because the patient did not show hypersalivation, and we used sugammadex for neuromuscular blockade reversal.

Finally, a regional or peripheral nerve block, either after anesthesia induction or at the end of surgery, is necessary for postoperative pain control and for prevention of postoperative disruption of surgical site due to continuous involuntary movements, cognitive dysfunction, and dyskinesia $[11,16]$.

In conclusion, VLI without neuromuscular blockade can be performed safely in awake HD patients with noncooperation if propofol sedation and topical anesthesia of the oral cavity are sufficient to prevent the gag reflex. The TIVA using propofol and remifentanil can be applied without any postoperative aggravation of $\mathrm{HD}$ symptoms and new postoperative neuropsychiatric symptoms if the surgical time is short and the psychotropic medication is re-administered as soon as possible after surgery. Rocuronium is recommended for neuromuscular block with monitoring of the TOF ratio, because it can be fully reversed with sugammadex successfully.

\section{CONFLICT OF INTEREST}

No potential conflict of interest relevant to this article was reported.

\section{REFERENCES}

1. McColgan P, Tabrizi SJ. Huntington's disease: a clinical re- view. Eur J Neurol 2018;25:24-34.

2. Nguyen PT, Meeks D, Liotiri D. Anaesthesia and orphan disease: airway and anaesthetic management in Huntington's disease. BMJ Case Rep 2017;2017:bcr2017221354.

3. Roos RA. Huntington's disease: a clinical review. Orphanet J Rare Dis 2010;5:40.

4. Kivela JE, Sprung J, Southorn PA, Watson JC, Weingarten TN. Anesthetic management of patients with Huntington disease. Anesth Analg 2010;110:515-23.

5. Haimov-Kaldess I, Haim D, Garfunkel A. General anesthesia for dental treatment in a patient with huntington's chorea. Compend Contin Educ Dent 2016;37:22-6.

6. Batra A, Sahni N, Mete UK. Anaesthetic management of a patient with Huntington's chorea undergoing robot-assisted nephron-sparing surgery. Indian J Anaesth 2016;60:866-7.

7. Sriganesh K, Saini J. Exacerbation of involuntary movements after propofol anesthesia in a patient with Huntington disease. J Neurosurg Anesthesiol 2013;25:212-4.

8. MacPherson P, Harper I, MacDonald I. Propofol and remifentanil total intravenous anesthesia for a patient with Huntington disease. J Clin Anesth 2004;16:537-8.

9. Nagele $P$, Hammerle AF. Sevoflurane and mivacurium in a patient with Huntington's chorea. Br J Anaesth 2000;85:3201.

10. Kang JM, Chung JY, Han JH, Kim YS, Lee BJ, Yi JW. Anesthetic management of a patient with Huntington's chorea -A case report-. Korean J Anesthesiol 2013;64:262-4.

11. Khan MH, Banerjee A. Anaesthesia and orphan disease: sugammadex in a patient with Huntington's disease undergoing thyroid lobectomy. Eur J Anaesthesiol 2012;29:593-5.

12. Cangemi CF Jr, Miller RJ. Huntington's disease: review and anesthetic case management. Anesth Prog 1998;45:150-3.

13. Saft C, Andrich J, Fälker M, Gauda S, Küchler S, Woitalla D, et al. No evidence of impaired gastric emptying in early Huntington's disease. PLoS Curr 2011;3:RRN1284.

14. Spooner B, Eagland K. The use of rocuronium and sugammadex in Huntingdon's disease. Anaesth Cases 2013;1:47-9.

15. White T, Neustein S. Monitored anesthesia care for a patient with advanced Huntington's chorea. Middle East J Anaesthesiol 2013;22:185-6.

16. Torrillo TM, Rosenblatt MA. Ultrasound-guided interscalene catheters performed under general anesthesia in a patient with Huntington's disease. Minerva Anestesiol 2010;76:6458. 